\title{
BMJ Open Likelihood of death among hospital inpatients in New Zealand: prevalent cohort study
}

\author{
Merryn Gott, ${ }^{1}$ Joanna Broad, ${ }^{2}$ Xian Zhang, ${ }^{2}$ Lene Jarlbaek, ${ }^{3}$ David Clark ${ }^{4}$
}

To cite: Gott M, Broad J, Zhang $X$, et al. Likelihood of death among hospital inpatients in New Zealand: prevalent cohort study. BMJ Open 2017;7:e016880. doi:10.1136/ bmjopen-2017-016880

- Prepublication history for this paper is available online. To view these files, please visit the journal online (http://dx.doi. org/10.1136/bmjopen-2017016880).

Received 19 March 2017 Revised 29 August 2017 Accepted 28 September 2017

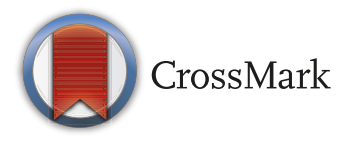

${ }^{1}$ The School of Nursing, The University of Auckland, Auckland, New Zealand ${ }^{2}$ Freemasons Department of Geriatric Medicine, The University of Auckland, Auckland, New Zealand ${ }^{3}$ Department of Clinical Research, The Danish Knowledge Centre for Rehabilitation and Palliative Care, University of Southern Denmark, Odense, Denmark ${ }^{4}$ School of Interdisciplinary Studies, University of Glasgow, Glasgow, UK

Correspondence to Professor Merryn Gott; m.gott@auckland.ac.nz

\begin{abstract}
Objectives (1) To establish the likelihood of dying within 12 months for a cohort of hospital inpatients in New Zealand (NZ) on a fixed census date; (2) to identify associations between likelihood of death and key sociodemographic, diagnostic and service-related factors and (3) to compare results with, and extend findings of, a Scottish study undertaken for the same time period and census date. National databases of hospitalisations and death registrations were used, linked by unique health identifier.
\end{abstract}

Participants 6074 patients stayed overnight in NZ hospitals on the census date (10 April 2013), $40.8 \%$ of whom were aged $\geq 65$ years; $54.4 \%$ were women; $69.1 \%$ of patients were NZ European; $15.3 \%$ were Maori; $7.6 \%$ were Pacific; $6.1 \%$ were Asian and $1.9 \%$ were 'other'. Setting All NZ hospitals.

Results $14.5 \%$ patients ( $n=878$ ) had died within 12 months: $1.6 \%$ by 7 days; $4.5 \%$ by 30 days; $8.0 \%$ by 3 months and $10.9 \%$ by 6 months. In logistic regression models, the strongest predictors of death within 12 months were: age $\geq 80$ years $(\mathrm{OR}=5.52(95 \% \mathrm{Cl} 4.31$ to 7.07)); a history of cancer ( $\mathrm{OR}=4.20$ (3.53 to 4.98)); being Māori ( $O R=1.62(1.25$ to 2.10)) and being admitted to a medical specialty, compared with a surgical specialty ( $\mathrm{OR}=3.16(2.66$ to 3.76$)$ ).

Conclusion While hospitals are an important site of end of life care in NZ, their role is less significant than in Scotland, where $30 \%$ of an inpatient cohort recruited using similar methods and undertaken on the same census date had died within 12 months. One reason for this finding may be the extended role of residential long-term care facilities in end of life care provision in NZ.

\section{BACKGROUND}

New Zealand (NZ), in line with other resource rich countries, is facing an unprecedented demand for palliative care within the short to medium term. Largely as a result of rapid population ageing, deaths in NZ are estimated to increase by $48 \%$ by $2038 .{ }^{1}$ Research conducted nationally has established that the acute hospital is a significant site of palliative care management with approximately one in five inpatients meeting Gold Standards Framework prognostic criteria for palliative care need, ${ }^{2}$ of whom approximately two-thirds will have died within 12 months $^{3}$
Strengths and limitations of this study

- First national picture of deaths among a cohort of inpatients present on one night in New Zealand (NZ) hospitals and close replication of a Scottish study undertaken on the same census date.

- Additional variables modelled for the first timeethnicity, admission type and history of main hospital-based diagnoses.

- Only those variables collected by the NZ Ministry of Health could be included.

- History of the various conditions, including cancer, based only upon diagnoses from hospitalisations occurring since 2004, so it does not include conditions managed entirely within primary care or hospitalisations prior to 2004 .

It is within this context that there has been increased interest nationally in hospital-based interventions to support improved palliative and end of life care management, including advance care planning and workforce capacity building. ${ }^{4}$ Policy-makers have also been looking internationally to identify innovations adopted in other countries facing similar challenges. However, international comparisons are limited by a lack of understanding of the comparability of patterns of service use at end of life. For example, previous estimates of the prevalence of palliative care needs among hospital inpatients range from $9 \%$ in Belgium ${ }^{6}$ to $17 \%$ in South Africa, ${ }^{7} 20 \%$ in NZ, ${ }^{2} 21 \%-36 \%$ in England ${ }^{8} 9$ and $35 \%$ in Australia. ${ }^{10}$ Moreover, methods adopted in these studies differed, as did definitions of 'palliative care need' and no study took a whole country approach.

A 2010 study addressed some of these deficits by reporting that $29 \%$ of a cohort of Scottish hospital inpatients on a selected census date died within 12 months. ${ }^{11}$ Factors associated with the likelihood of dying included being >85 years, living in an area of high deprivation, and being admitted under a medical specialty (rather than surgical). The study was replicated in both Scotland and 
NZ for the same 2013 census date. The 2013 Scottish study produced very similar results to the original Scottish study (30\% dying within 12 months), supporting the robustness of the findings. ${ }^{12}$ Replicating and extending the study within $\mathrm{NZ}$ was identified as helpful for national planning, supporting clinicians to respond appropriately to potential palliative care need, and in order to build a better understanding of comparative service use among people in the last year of life, internationally.

\section{AIMS}

- To identify the proportion of a cohort of NZ public hospital inpatients dying within 12 months of a given census date.

- To identify associations between likelihood of death and key sociodemographic, diagnostic and service-related factors.

- To compare results with, and extend findings of, a Scottish study undertaken for the same time period and census date.

\section{Materials and methods}

The NZ Ministry of Health provided data for all publicly funded hospitalisations (hospitalisations in public acute hospital and publicly funded private surgical hospitals) in NZ over the period 20 January 2004 to 10 April 2013 for people in hospital overnight on the census date of 10 April 2013. Data included: demographic information (age, gender, deprivation of area of residence and prioritised self-identified ethnicity); hospitalisation (admission date and type, discharge date and type, length of stay, specialty of the attending physician at discharge and diagnosis) and date of death if it occurred during this hospital stay. National death registrations data provided date of death for all deaths within 12 months of census date, and linked by the Ministry of Health to hospital stay record through unique national health identifiers. Self-reported ethnicity was prioritised, whereby those identifying with more than one ethnic group are classified first to Māori, then to Pacific or Asian ethnicity. ${ }^{13}$

For each hospitalisation, the patient's primary diagnosis was classified using ICD-10 chapter. To identify prior history of five selected diagnostic groups (cancer, circulatory diseases, respiratory diseases, injury/poisoning/ other consequences of external causes, and digestive diseases), all diagnoses coded for all prior hospital admissions over the period 20 January 2004 to 10 April 2013 (including the index stay) of each patient in the index cohort were reviewed and coded with a binary indicator ( $1=$ yes, $0=$ no). The measure of deprivation used was the NZ Index of Deprivation 2006, ${ }^{14}$ an area-based deprivation score grouping the $\mathrm{NZ}$ population into 10 deciles based on place of residence, with decile 1 representing the $10 \%$ least deprived areas in NZ and decile 10 the most deprived, collapsed into quintiles.

Three multiple logistic regressions (described below) were used for modelling to investigate associations between potential predictor variables and mortality at 12 months. The c-index (the area under receiver-operating curve) was used to assess the ability of the models to discern those who died from those who were alive at 12 months.

In model 1, the response variable was whether the patient died within 12 months. There were four predictor variables in the model: gender (woman as referent), age group $(0-<15,15-<60,60-64,65-69,70-74,75-79,80-84$ and $85+$ years; $15-<60$ years was used as referent), deprivation quintile $(\mathrm{Q} 1$ is the most deprived and $\mathrm{Q} 5$ is the least deprived; Q5 was used as referent) and specialty (medicine, surgery or procedure; surgery was used as referent). Of the 6074 patients recorded as resident in NZ hospitals on the census date, 6029 patients were included for modelling; patients for whom deprivation status was not available were excluded $(n=45)$.

Model 2 was developed from model 1 by adding three predictor variables: admission type (acute, arranged or waitlisted; acute was used as referent); prioritised ethnicity (European, Māori, Pacific, Asian and other; European was used as referent) and history of cancer.

Model 3 was further developed from model 2 by adding four other diagnostic history groupings: circulatory diseases, respiratory diseases, injury/poisoning/other consequences of external causes, and digestive diseases.

MS Excel 2010 and SAS V.9.4 were used for all analyses.

\section{RESULTS}

In total, 6074 publicly funded patients stayed overnight in NZ hospitals on the census date, $46 \%$ of whom were men and $54 \%$ women. Forty-one per cent were aged $\geq 65$ years; $17 \%$ were aged $>80$ years (table 1 ). Sixty-eight per cent were acute admissions, $18 \%$ arranged admissions and $14 \%$ waitlisted admissions. Based on NZ death registration records, $878(14.5 \%)$ patients died during the 12 months following the census date: $1.6 \%$ by 7 days and $10.9 \%$ by 6 months. One hundred and thirty patients $(2.1 \%)$ died in hospital during the index stay and these deaths accounted for $14.8 \%$ of all deaths within the 12-month follow-up period.

On the census date, the two most deprived population quintiles (Q1 and $\mathrm{Q} 2)$ contributed $50 \%$ of hospitalisations, whereas the two least deprived quintiles $(\mathrm{Q} 4$ and Q5) contributed only 29\% of hospitalisations. At 12 months from the census date, the two most deprived quintiles contributed $51 \%$ of deaths and the two least deprived quintiles contributed $27 \%$ of deaths. A much greater proportion of those with a history of cancer had died within 12 months at $33 \%$, when compared with $9 \%$ without a history of cancer. Ten per cent of those with a history of cancer had died within 30 days of their admission (table 2). Patients with one of the five chosen diagnostic groups (cancer, circulatory diseases, respiratory diseases, injury/poisoning/other consequences of external causes, and digestive diseases) as primary diagnosis in the index hospital stay contributed to $69 \%$ of all 


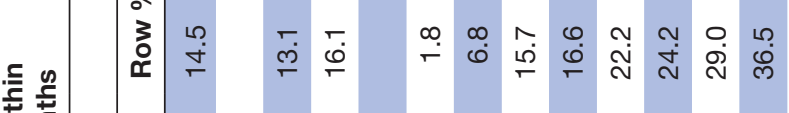

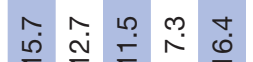

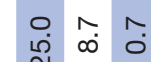

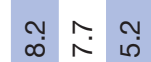

ウ.

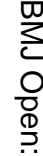

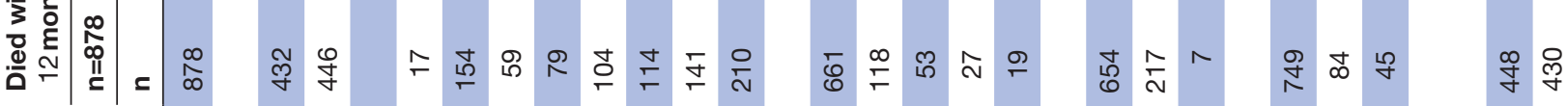

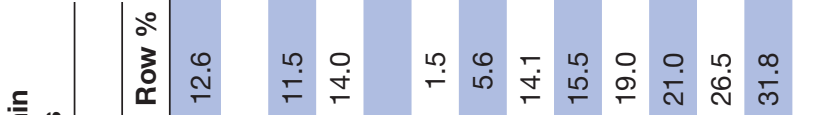

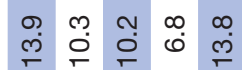

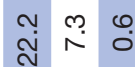

\begin{tabular}{ccc}
- & $\infty$ & $\infty$ \\
\hdashline & 0 & $\oplus$
\end{tabular}

๘.

焉䍃

彭鰝

8

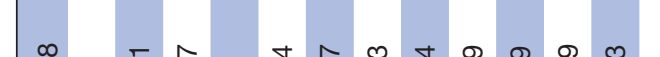

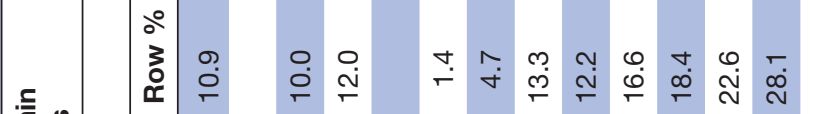

高

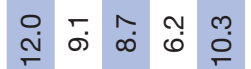

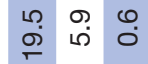

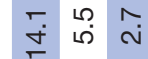

๑ึ

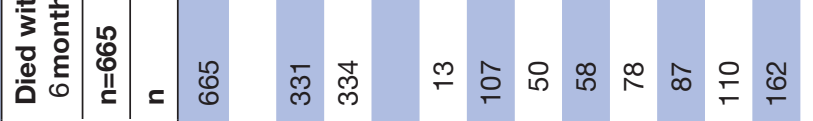

央

๙ 8 ก

ஜ్లి ద్లె

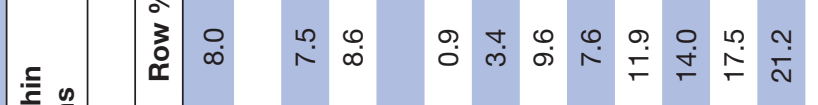

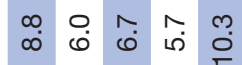

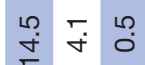

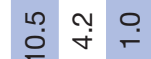

우 둥

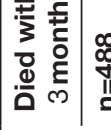

产

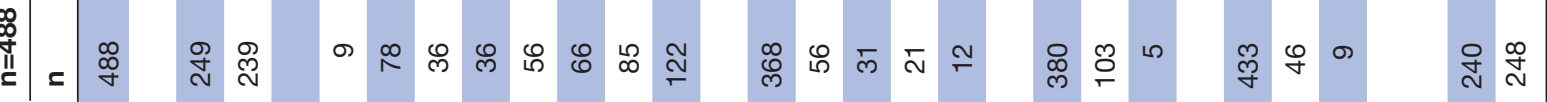

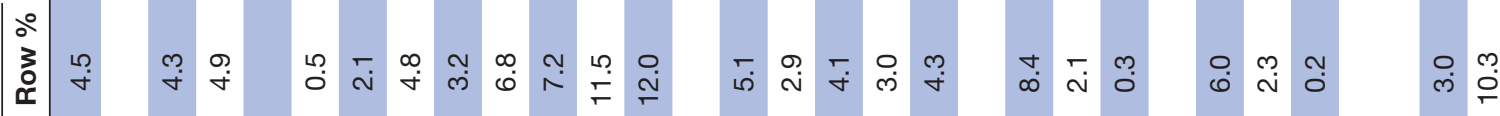

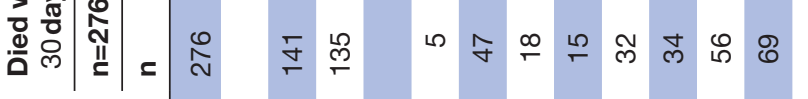

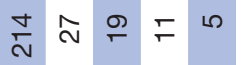

กิ กั ก

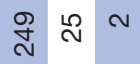

$\stackrel{\leftarrow}{\stackrel{一}{\mathscr{m}}}$

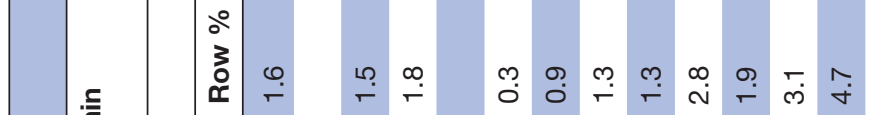

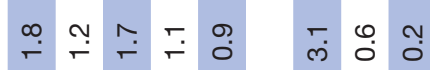

$\stackrel{\text { Nิ }}{\stackrel{\infty}{0}}$

$\stackrel{m}{\Gamma} \stackrel{\infty}{\sim}$

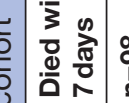

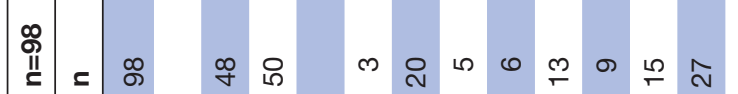

A $\leftarrow \infty+$

$\bar{\infty} \stackrel{\Gamma}{\circ} \sim$

$\infty \varnothing 0$

ฮิ

离

б.

ர்:

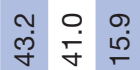

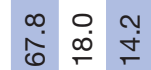

$\stackrel{\sim}{\stackrel{\infty}{\sim}} \stackrel{\infty}{\stackrel{N}{N}}$

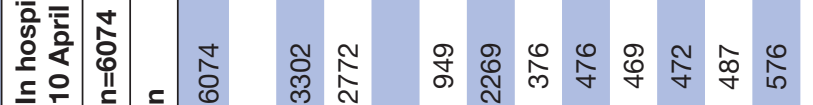

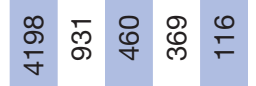

त్

둗

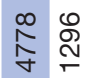
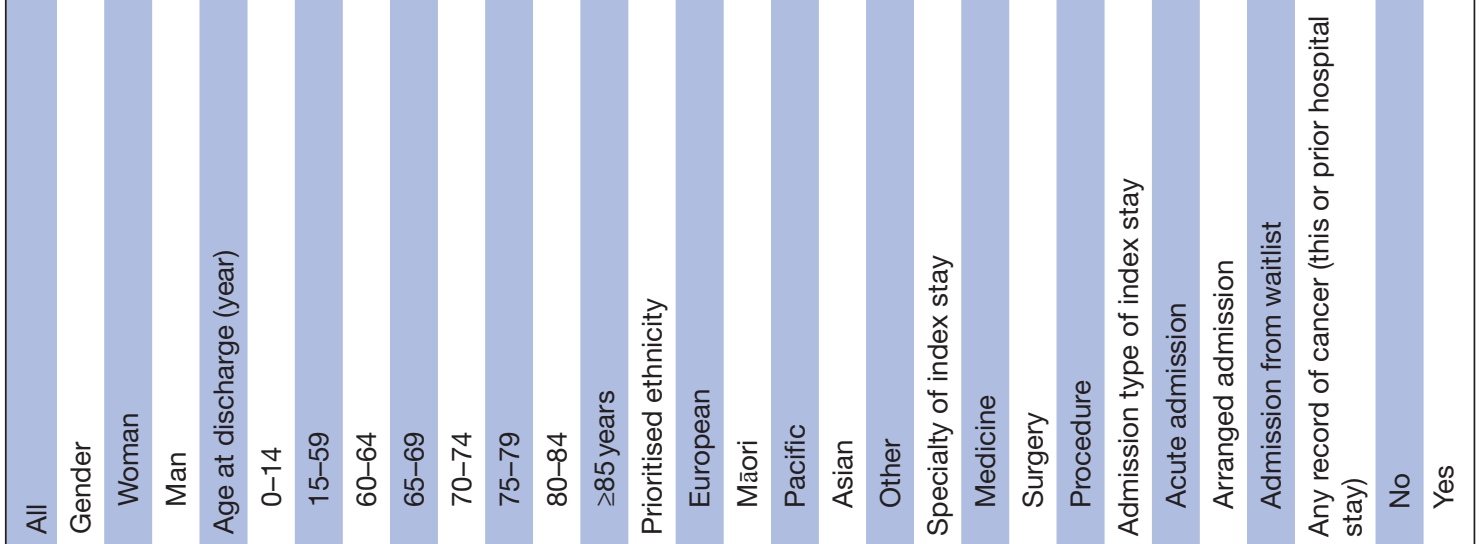


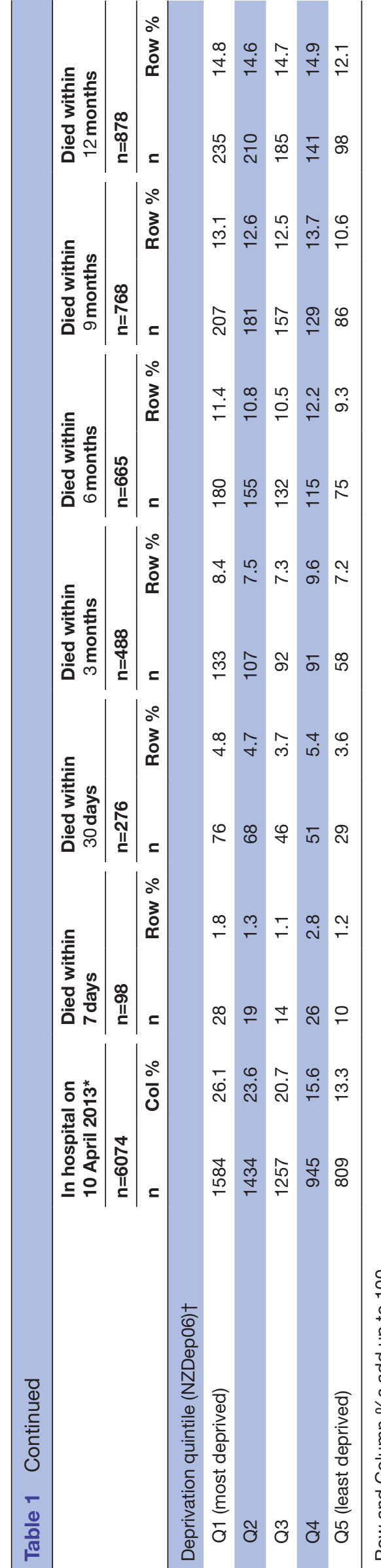

deaths (602 of 878 deaths within 12 months of census date, figure 1$)$.

Model 1 showed that mortality rose steeply with age, for example, patients $>85$ years years were far more likely to die (OR=5.52 (95\% CI 4.31 to 7.07)), compared with patients between 15 and 59 years (table 3). Patients in the most deprived quintile were more likely to die $(\mathrm{OR}=1.54$ (1.17 to 2.02)) compared with patients in the least deprived quintile. Patients admitted to a medical specialty were also more likely to die $(\mathrm{OR}=3.16$ (2.66 to 3.76)) compared with patients admitted to a surgical specialty. Those admitted for a procedure were less likely to die $(\mathrm{OR}=0.26$ (0.12 to 0.57$))$ than those admitted to a surgical specialty. The high c-index $(0.79)$ indicates that almost $80 \%$ of the variability was explained by the model.

Model 2 showed that Māori were more likely to die $(\mathrm{OR}=1.62$ (1.25 to 2.10)), than Europeans, as were patients with a history of cancer ( $\mathrm{OR}=4.20$ (3.53 to 4.98)) compared with those without it. Waitlisted patients were less likely to die $(\mathrm{OR}=0.34$ (0.24 to 0.48$))$ compared with those who came in acutely (table 3 ). Including the additional variables improved the c-index considerably to 0.84 . The addition of previous hospital diagnoses in model 3 showed patients with a history of circulatory diseases were more likely to die $(\mathrm{OR}=1.34$ (1.09 to 1.64)) compared with those without it (table 3 ). Those with a history of respiratory diseases were more likely to die $(\mathrm{OR}=1.82$ (1.53 to 2.16$))$ versus those without it. Those with a history of digestive diseases were more likely to die $(\mathrm{OR}=1.51$ (1.26 to 1.80$))$ versus those without it. After adjusting for these additional diagnostic groups, history of cancer remained highly significant $(\mathrm{OR}=3.92$ (3.29 to $4.67)$ ). The c-index of 0.85 indicates that the model barely improved when including the four additional diagnostic history variables.

\section{DISCUSSION}

This study identified that $14.5 \%$ of patients resident in NZ hospitals on one day had died within 12 months. This proportion is much lower than that reported by a study of Scottish inpatients conducted using the same method and census date, where $30 \%$ had died within 12 months. ${ }^{12}$ Similarly, while in Scotland $8 \%$ of patients died during the index admission (representing $32 \%$ of all deaths in this cohort within the 12-month period), the figures for NZ were much lower at $2 \%$, accounting for $15 \%$ of all deaths in the cohort over 12 months.

Reasons for the much lower mortality in NZ compared with Scotland are not easily determined, but several interpretations warrant consideration. One important difference to note is the younger age of the NZ inpatient hospital population compared with the Scottish inpatient hospital population-73\% of the Scottish inpatients were $\geq 60$ years and $32 \%$ were $\geq 80$ years, ${ }^{12}$ while just $42 \%$ of the NZ inpatients were aged $\geq 60$ years, of whom $17 \%$ were aged $\geq 80$ years (see table 1 ). The younger age in NZ's cohort means fewer deaths would be expected and 


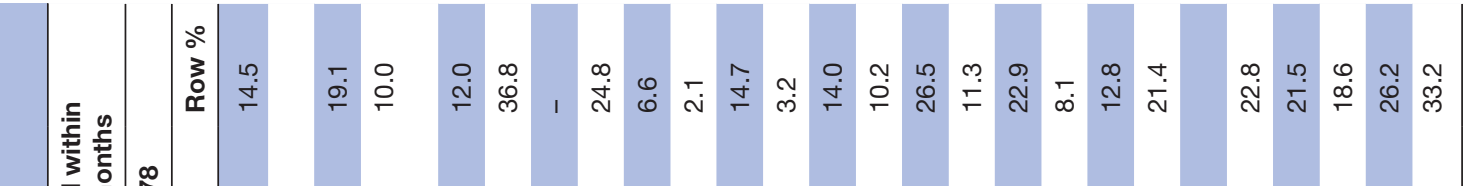

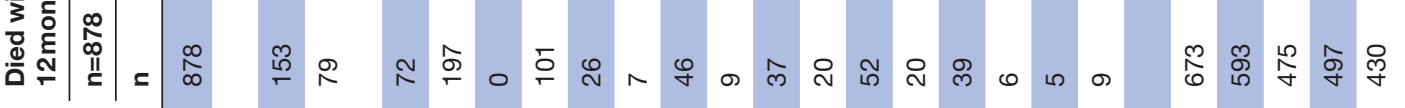

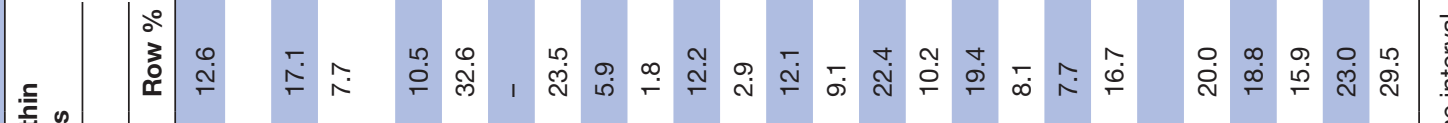

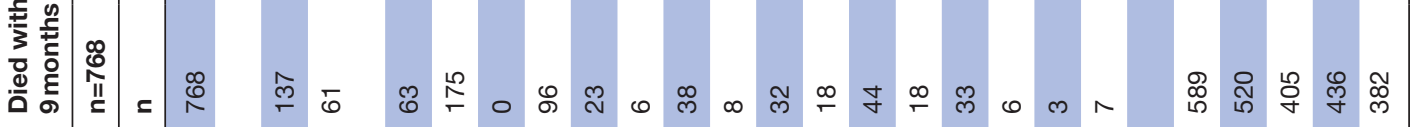

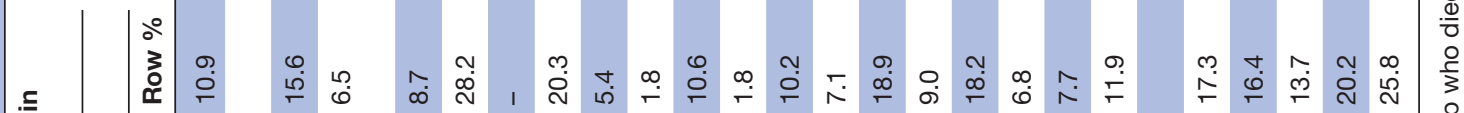

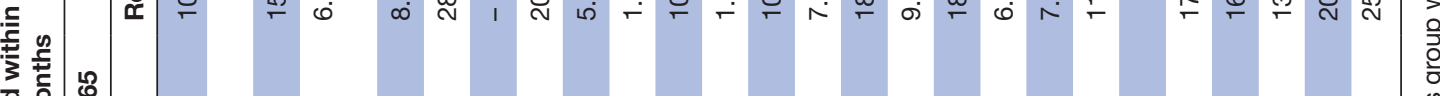

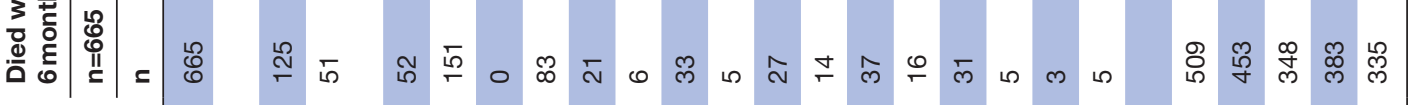

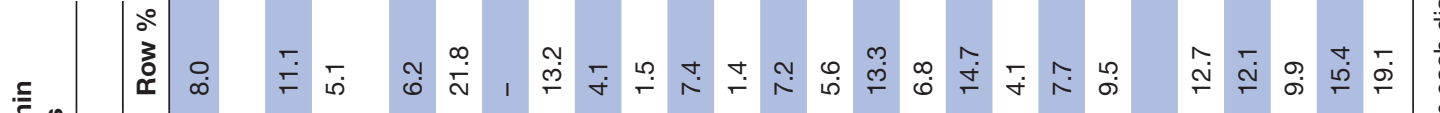

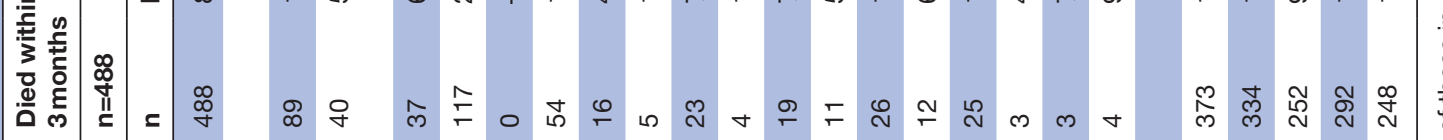

(2)

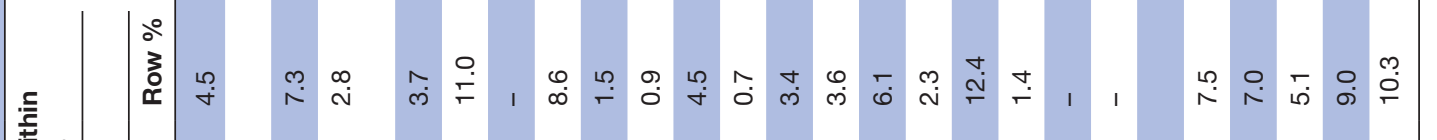

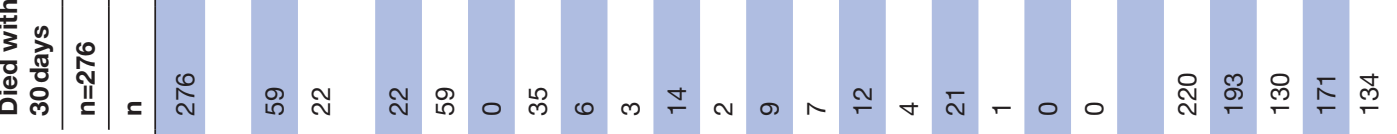

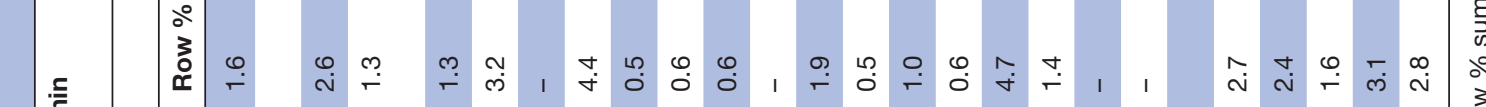

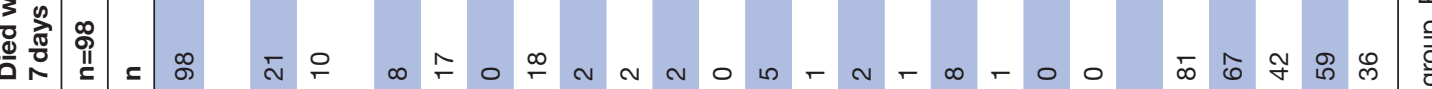

б. 


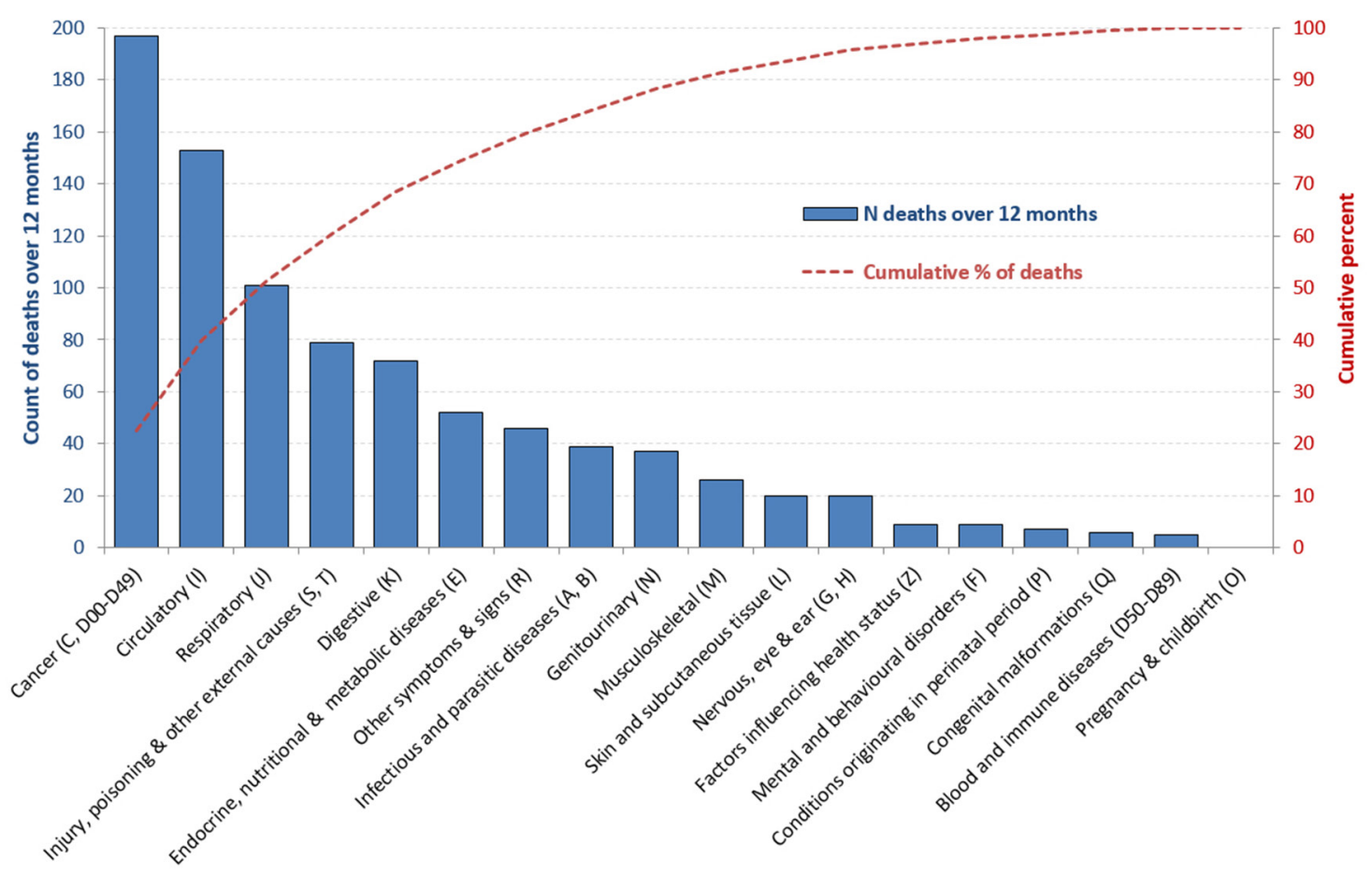

Primary diagnosis recorded for index hospital stay

Figure 1 Deaths within 12 months of census date by primary diagnosis of hospitalisation.

reflects the fact that the Scottish and NZ acute hospital populations are different.

There is also evidence that hospitals represent a much more significant place for end of life care and death in Scotland than in NZ. In Scotland, 59\% of deaths occur in hospital, $18 \%$ in residential long-term care and $23 \%$ in other settings, which includes home. ${ }^{15}$ In NZ, a much lower percentage die in hospital at $34 \%$, and a much higher proportion die in residential long-term care at $31 \%$ and in other settings including home, which account for $35 \%$ of all deaths. ${ }^{15}$ The argument that, in NZ, highlevel residential long-term care facilities may act as 'de facto' hospices is also supported by a recent study by Connolly et al. ${ }^{16}$

While our findings confirm that the proportion of a prevalence sample of hospital inpatients dying within 12 months in NZ is lower than that in Scotland, the fact that $14.5 \%$ do so is not insignificant. Indeed, when considered alongside our previous NZ research showing that in a cross-sectional inpatient cohort, one in five meets criteria for palliative care needs, ${ }^{23}$ this study helps build a picture of the acute hospital as a major site of end of life care delivery.

The consistency of predictors of the likelihood of dying within 12 months between NZ and Scotland when the same variables are modelled is interesting, although not unexpected. Indeed, the finding that age is the strongest predictor of death within 12 months reflects the situation in many resource-rich countries where dying in advanced age is now the norm. ${ }^{17}$ Similarly, the association between living in an area of high deprivation and mortality rates is well known and holds true for both NZ and Scotland, ${ }^{18-20}$ although it is important to note that deprivation of place of residence became non-significant in the NZ models once diagnostic history and ethnicity were adjusted for.

The NZ study also extended the Scottish study by modelling additional variables. This identified that inpatients with a history of cancer were more likely to die than those without a history of cancer, as were those whose admission was acute or who were Māori. This is important because deprivation no longer becomes an important predictor, suggesting that the association of deprivation with death within 12 months is related more to ethnicity (and related factors not measured) than to deprivation itself. These findings confirm known associations between cancer and mortality rates, the nature of acute admissions and the higher rate of chronic conditions, including cancer, among Māori compared with non-Māori. ${ }^{21} 22$

\section{Strengths and limitations}

This study provides a national picture of deaths among a cohort of inpatients present on one night in NZ hospitals. It closely replicates a Scottish study undertaken on the same census date (although unlike the Scottish study it did not exclude obstetric patients). It extends that study by modelling additional variables-ethnicity, admission type and history of main hospital-based diagnoses. However, certain limitations must be acknowledged. History of the various conditions, including cancer, is based only upon diagnoses from hospitalisations occurring since 2004, so it does not include conditions managed entirely within primary care, or hospitalisations prior to 2004. NZDep, as 
Table 3 Comparison of three predictive models for death within 12 months

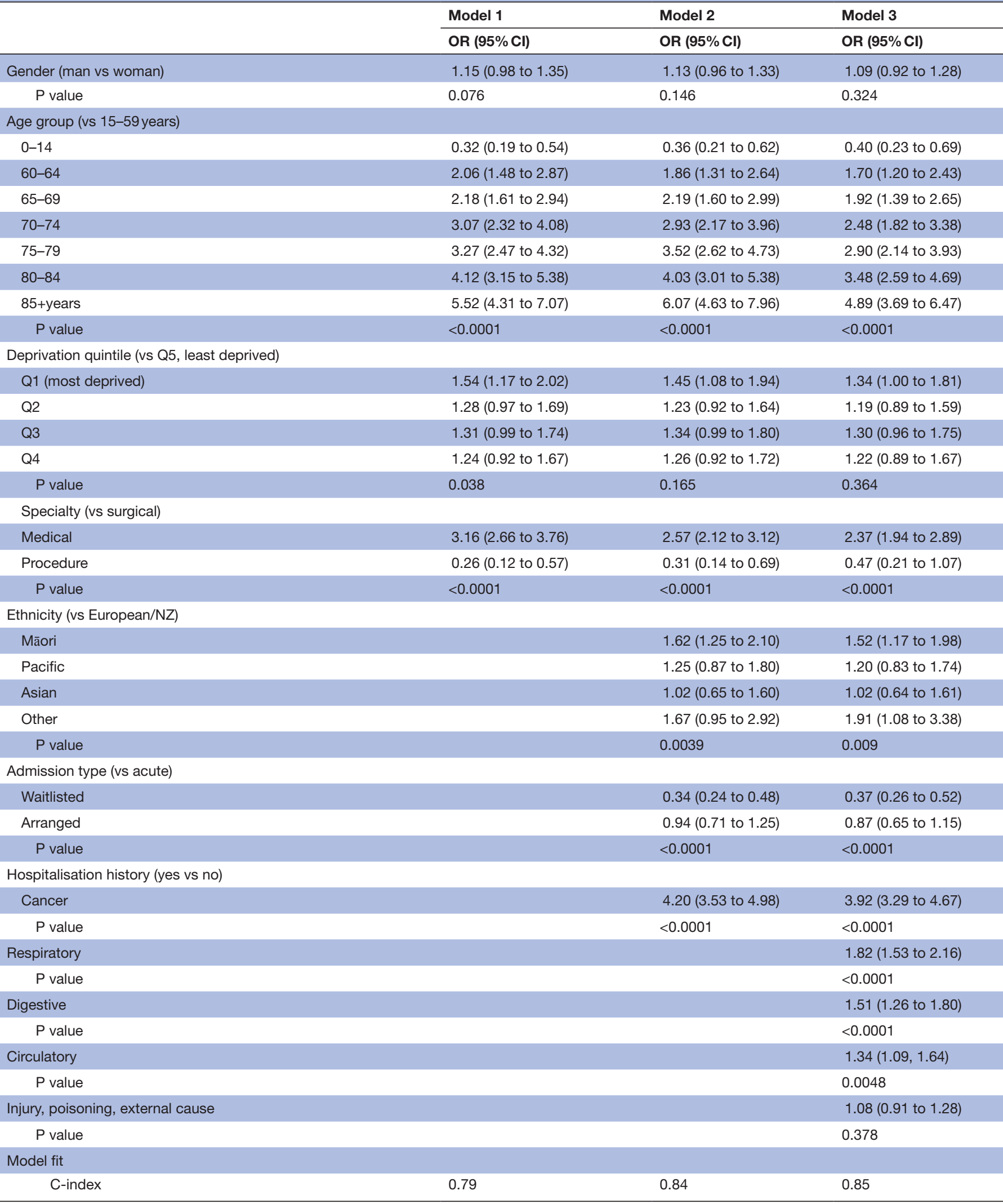

45 people were omitted from all models because of missing data for deprivation.

a measure of deprivation for older people, is a poor indicator of a lifetime deprivation, especially for those living in long-term care, but the measure is what is available. The problems of length-biased sampling inherent in a cohort assembled from a cross-sectional study mean that patients experiencing longer hospital stays are over-represented 
in both studies. Our study population was of all those in hospital on a particular date and not of admissions on that date. Finally, it was possible to include only those variables collected by the Ministry of Health in our modelling.

\section{CONCLUSION}

This study compared the likelihood of death of a cohort of NZ hospital inpatients with a cohort of Scottish hospital inpatients from the same census date over a 12-month period. From the NZ cohort, $14.5 \%$ had died within a 12 month period-half that of the Scottish cohort (30\%). While the reasons underpinning this finding warrant further research, overall the study points to interesting variations in health service usage between countries and confirms the utility of conducting international comparative studies, of which there are few within palliative care.

Contributors MG, made a substantial contribution to study design and interpretation, drafted the majority of the paper and revised following coauthor feedback, and acts as guarantor for the paper. JB, made a substantial contribution to study design and interpretation, led the analysis, had input into the paper and approved the final version. XZ, contributed to study design, undertook the analysis, and reviewed and approved the final paper. LJ and DC, contributed to study design and interpretation, provided input into the paper, and approved the final version.

Competing interests None declared.

Ethics approval University of Auckland Human Participants Ethics Committee (ref: 02/11/2015).

Provenance and peer review Not commissioned; externally peer reviewed.

Data sharing statement Access to data analysed for the purposes of this study is via the NZ Ministry of Health.

Open Access This is an Open Access article distributed in accordance with the Creative Commons Attribution Non Commercial (CC BY-NC 4.0) license, which permits others to distribute, remix, adapt, build upon this work non-commercially, and license their derivative works on different terms, provided the original work is properly cited and the use is non-commercial. See: http://creativecommons.org/ licenses/by-nc/4.0/

(c) Article author(s) (or their employer(s) unless otherwise stated in the text of the article) 2017. All rights reserved. No commercial use is permitted unless otherwise expressly granted.

\section{REFERENCES}

1. McLeod H. The need for palliative care in New Zealand: Technical Report prepared for the Ministry of Health, 2016. http://centraltas. co.nz/health-of-older-people/tools-and-guidance/. (accessed 2 Dec 2016).

2. Gott M, Frey R, Raphael D, et al. Palliative care need and management in the acute hospital setting: a census of one New Zealand Hospital. BMC Palliat Care 2013;12:1-8.
3. O'Callaghan A, Laking G, Frey R, et al. Can we predict which hospitalised patients are in their last year of life? A prospective cross-sectional study of the Gold Standards Framework Prognostic Indicator Guidance as a screening tool in the acute hospital setting. Palliat Med 2014;28:1046-52.

4. NHS England. Transforming end of life care in acute hospitals: The route to success 'how to' guide. Leeds: NHS England, 2015.

5. Ministry of Health. Palliative care review. Wellington: Ministry of Health. Forthcoming.

6. Desmedt MS, de la Kethulle YL, Deveugele MI, et al. Palliative inpatients in general hospitals: a one day observational study in Belgium. BMC Palliat Care 2011:10:1-8.

7. van Niekerk L, Raubenheimer PJ. A point-prevalence survey of public hospital inpatients with palliative care needs in Cape Town, South Africa. S Afr Med J 2014;104:138-41.

8. Gott MC, Ahmedzai SH, Wood C. How many inpatients at an acute hospital have palliative care needs? Comparing the perspectives of medical and nursing staff. Palliat Med 2001;15:451-60.

9. Gardiner C, Gott M, Ingleton C, et al. Extent of palliative care need in the acute hospital setting: a survey of two acute hospitals in the UK. Palliat Med 2013;27:76-83.

10. To TH, Greene AG, Agar MR, et al. A point prevalence survey of hospital inpatients to define the proportion with palliation as the primary goal of care and the need for specialist palliative care. Intern Med J 2011;41:430-3.

11. Clark D, Armstrong M, Allan A, et al. Imminence of death among hospital inpatients: Prevalent cohort study. Palliat Med 2014;28:474-9.

12. Clark D, Schofield L, Graham FM, et al. Likelihood of death within one year among a national cohort of hospital inpatients in Scotland. $J$ Pain Symptom Manage 2016;52:e2-e4.

13. Callister P, Didham R, Potter D, et al. Measuring ethnicity in New Zealand: developing tools for health outcomes analysis. Ethn Health 2007;12:299-320.

14. Salmond C, Crampton P, Atkinson J. NZDep2006: Index of Deprivation. Wellington: Ministry of Health, 2007.

15. Broad JB, Gott M, Kim H, et al. Where do people die? An international comparison of the percentage of deaths occurring in hospital and residential aged care settings in 45 populations, using published and available statistics. Int $J$ Public Health 2013;58:257-67.

16. Connolly MJ, Broad JB, Boyd M, et al. Residential aged care: the de facto hospice for New Zealand's older people. Australas J Ageing 2014;33:114-20.

17. Cohen J, Gott M. Dying in place in old age: public health challenges. Palliative care for older people: A public health perspective. Oxford: Oxford University Press, 2015:107-19.

18. Richardson K, Blakely T, Young J, et al. Do ethnic and socioeconomic inequalities in mortality vary by region in New Zealand? An application of hierarchical Bayesian modelling. Soc Sci Med 2009;69:1252-60.

19. Pearce JR, Richardson EA, Mitchell RJ, et al. Environmental justice and health: the implications of the socio-spatial distribution of multiple environmental deprivation for health inequalities in the United Kingdom. Transactions of the Institute of British Geographers 2010;35:522-39.

20. Everson-Rose SA, Skarupski KA, Barnes LL, et al. Neighborhood socioeconomic conditions are associated with psychosocial functioning in older black and white adults. Health Place 2011;17:793-800.

21. Ministry of Health. Tatau Kahukura: Māori Health Chart Book. 2015. 3rd edn. Wellington: Ministry of Health, 2015.

22. Tobias M, Blakely $\mathrm{T}$, Matheson $\mathrm{D}$, et al. Changing trends in indigenous inequalities in mortality: lessons from New Zealand. Int $J$ Epidemiol 2009;38:1711-22. 